\title{
THEORETICAL MODEL FOR THE LATITUDE DEPENDENCE OF THE THERMO SPHERIC ANNUAL AND SEMIANNUAL VARIATIONS
}

\author{
H. G. MAYR \\ H. VOLLAND
}

JÛ́NE 1972

CSSFC

GODDARD SPACE FLIGHT CENTER

GREENBELT MARYLAND

(NASA-TH-X-65961) THEORETICAL MODEL FOR
THE LATITUDE DEPENDENCE OF THE

N72-28357

THERMOSPHERIC ANNUAL AND SEMIANNUAL

VARIATIONS

H.G. Mayr, et al (NASA)

$1972.29 \mathrm{p}$ 
THEORETICAL MODEL FOR THE LATITUDE DEPENDENCE OF THE THERMOSPHERIC ANNUAL AND SEMIANNUAL VARIATIONS

\author{
by \\ H. G. Mayr \\ Thermosphere and Exosphere Branch \\ Goddard Space Flight Center \\ Greenbelt, Maryland \\ and \\ H. Volland \\ Astronomical Institutes \\ University of Bonn \\ Bonn, W. Germany
}

June 1972

Goddard Space Flight Center

Greenbelt, Maryland 


\section{CONTENTS}

Page

ABSTRACT ........................ v

1. INTRODUCTION $\ldots \ldots \ldots \ldots \ldots \ldots \ldots \ldots \ldots \ldots \ldots \ldots \ldots$

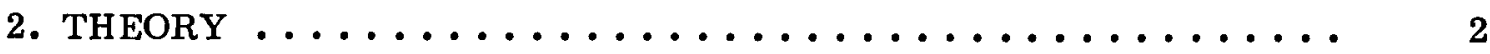

3. INPUT PARAMETERS $\ldots \ldots \ldots \ldots \ldots \ldots \ldots \ldots \ldots \ldots \ldots$

4. ANNUAL VARIATIONS ..................... 12

5. SEMIANNUAL VARIATION $\ldots \ldots \ldots \ldots \ldots \ldots \ldots \ldots \ldots \ldots$

6. CONCLUSION $\ldots \ldots \ldots \ldots \ldots \ldots \ldots \ldots \ldots \ldots \ldots \ldots \ldots \ldots \ldots \ldots$

ACKNOWLEDGEMENT ..................... 22

REFERENCES........................ 22

\section{ILLUSTRATIONS}

$\underline{\text { Figure }}$

$\underline{\text { Page }}$

1 Schematic diagram for the physical processes that influence the temperature and composition of the thermosphere .......

2 Schematic picture for the latitude dependence of the annual component of the various physical parameters with the important processes emphasized in circles . . . . . . . . . .

3 Relative amplitudes for the $\mathrm{O}-, \mathrm{He}-$, and $\mathrm{N}_{2}$-concentrations, the mass density $\rho$ and the gas temperature $\mathrm{Tg}$ in the annual component. The values are valid for the summer pole and are the coefficients of the spherical function $\mathrm{P}_{1}(\theta)=\cos \theta(\theta=$ polar distance) which describes the latitude dependence. Positive signs indicate positive, maximum values; negative signs indicate negative, minimum values in the northern summer hemisphere. Solid lines are taken from the $\mathrm{N}_{2}-\mathrm{O}$-model which describes correctly the energetics and thus the gas temperature. Dashed lines stem from the He-model which is energetically unrealistic corresponding to the assumption of diffusive equilibrium 
ILLUSTRATIONS-(continued)

$\underline{\text { Figure }}$

Page

3 for the major constituents $\mathrm{N}_{2}$ and $\mathrm{O}$ up to $500 \mathrm{~km}$. Note the

(cont.) large difference between the "diffusive equilibrium" solution and the "diffusion" solution for the gas temperature Tg as well as the small and nearly height independent amplitude of the mass density $\rho_{\ldots} \ldots \ldots \ldots \ldots \ldots \ldots \ldots \ldots \ldots$

4 Amplitudes from the thermospheric wind field in the annual component of the $\mathrm{N}_{2}-\mathrm{O}$-model. The maximum vertical velocities, W, are directed upward and are shown for the summer pole, the maximum meridional velocities, $U$, blowing toward the winter hemisphere are shown at the equator, and in the northern hemisphere the zonal winds, $V$, are eastward in winter and westward in summer with their maximum amplitudes at $45^{\circ}$ latitude. Dashed lines are for the O-component, solid lines are for the $\mathrm{N}_{2}$-component. Note that the difference between both constituents is particularly large for $\mathrm{W}$, but is also not negligible for $\mathrm{U} \ldots \ldots \ldots \ldots \ldots \ldots \ldots \ldots \ldots$

5 The $\mathrm{N}_{2}-\mathrm{O}$-model shown for different eddy diffusion coefficients. Note that all parameters are affected including $\mathrm{Tg} . \ldots . \ldots$. .

6 Relative amplitudes for the physical parameters of density and temperature of the semiannual variations. The values are valid for the poles and are the coefficients of the spherical harmonic $P_{2}$ $(\theta)$. Positive signs indicate at the poles maximum and minimum values during equinox and solstice, respectively. Negative signs indicate the opposite. The $\mathrm{N}_{2}-\mathrm{O}$-model and the He-model are differentiated with solid and dashed lines, respectively. Note the large difference between "diffusive equilibrium" solution (dashed line) and "diffusion" solution (solid line) for $\mathrm{Tg}$, and the height independent and very small amplitude of the mass density $\rho$.. 
THEORETICAL MODEL FOR THE LATITUDE DEPENDENCE OF THE THERMOSPHERIC ANNUAL AND SEMIANNUAL VARIATIONS

by

\author{
H. G. Mayr \\ Thermosphere and Exosphere Branch \\ Goddard Space Flight Center \\ Greenbelt, Maryland \\ and \\ H. Volland \\ Astronomical Institutes \\ University of Bonn \\ Bonn, W. Germany
}

\begin{abstract}
A three dimensional model for the annual and semiannual variations of the thermosphere is presented in which energy and diffusive mass transport associated with the global circulation are considered in a self consistent form. It is shown that these processes play a major role in the thermosphere dynamics and thus account for a number of temperature and composition phenomena such as

(a) The helium and oxygen bulges in the winter hemispheres at lower altitudes,

(b) The relatively large temperature variations $(\sim 26 \%)$ in the annual component exceeding by a factor of three those inferred from the satellite drag data and which would be much too large for a thermosphere in diffusive equilibrium,

(c) The 7\%-variation of the exospheric temperature at the poles in the semiannual component associated there with a significant depletion of oxygen and helium during the equinox;

(d) The nearly height independent and relatively small amplitude in the total mass density which is responsible for the fact that the latitude dependence of the semiannual effect has so far eluded observations from the satellite drag data.
\end{abstract}




\section{THEORETICAL MODEL FOR THE LATITUDE DEPENDENCE OF THE THERMOSPHERIC ANNUAL AND SEMIANNUAL VARIATIONS}

\section{INTRODUCTION}

The seasonal variations in the thermospheric density, as deduced from satellite drag data (Jacchia et al., 1966), have been of great concern for a long time. It should be expected that the annual and semiannual components are in a relation that corresponds approximately to the corresponding harmonic components of the solar heat input. Thus, energetically, a major problem emerged when it was observed that the semiannual effect appeared to be latitude independent (Cook, 1970) and that its magnitude seemed to be equal to that of the annual component.

The annual component of the thermosphere has so far been observed primarily from satellite drag data which were interpreted in terms of temperature variations (e.g. Jacchia and Slowey, 1967; Volland and Mayr, 1971). However, the existence of the winter anomaly in the $F_{2}$ region has led very early to the speculation (Johnson, 1964) that the annual component in the thermospheric wind field would decrease the oxygen content in the summer hemisphere, a hypothesis that was confirmed by Mayr and Volland (1971). The discovery of the winter helium bulge (Keating and Prior, 1968) brought further evidence for the significance of the large scale circulation to which it was attributed by Johnson and Gottlieb (1970).

Until recently all models have attributed the semiannual density variations to corresponding variations in the thermospheric temperature (Jacchia et al., 1966; Volland, 1969; Johnson, 1964; Newell, 1968).

Jacchia et al., (1966) associated temperatures to the observed density variations, Volland (1969), in following a suggestion by Newell (1968), explained the semiannual temperature amplitudes by waves leaking from the mesosphere up into the ther mosphere and Johnson (1964) proposed annually varying large scale meridional circulations which subtract more energy from the thermosphere during solstice than during equinox.

Cook (1967) proposed changes in the turbopause level to explain the semiannual variations at higher altitudes $(800 \mathrm{~km})$ where helium becomes the major constituent. This mechanism, however, which invokes variations in the eddy diffusion coefficient below $120 \mathrm{~km}$, and consequently would also change the relative concentration of atomic oxygen was examined by Harris and Priester (1969) 
and Cook (1969) who concluded that it would be inconsistent with the semiannual variations in the thermosphere up to $700 \mathrm{~km}$. In analyzing the semiannual effect in the $\mathrm{F}_{2}$ region Mayr and Mahajan (1971) concluded that there should be significant semiannual variations in the $(\mathrm{O}) /\left(\mathrm{O}_{2}\right)$ and $(\mathrm{O}) /\left(\mathrm{N}_{2}\right)$ ratios in the lower thermosphere with maxima occurring during equinox an "observation" that appeared to be consistent with the few reported rocket measurements. In explaining these variations in the neutral composition a circulation model was proposed by Mayr and Volland (1971)。 In this model the semiannual component of the meridional wind circulation was attributed to a primary energy source at high latitudes (presumed to be associated with the semiannual component in the occurrence of magnetic stroms) and to a much weaker secondary source at low latitudes (associated with the solar EUV heat input). Under the influence of this circulation, which was matched to agree with mesospheric wind measurements, atomic oxygen is redistributed semiannually such that during equinox $O$ is depleted at high latitudes (and to a much lesser degree at the equator) and enhanced at mid to low latitudes. Since this mechanism produces during equinox and at mid to low latitudes enhancements in the concentration of atomic oxygen without invoking temperature variations, it was concluded that the models that relate (at these latitudes) the semiannual density variations solely to temperature effects would probably overestimate the semiannual temperature amplitude. In a recent paper, Jacchia (1971) completely abandoned the temperature derivation from the semiannual effect in his model, stating that all difficulties in the description of the semiannual density variations could be removed if they were not related to temperature variations. Furthermore, Brinton and Mayr (1971) concluded from the small semiannual component in atomic hydrogen at mid latitudes that the semiannual variations in the exospheric temperature should be negligible there.

In combining all these evidences one might therefore suggest that a significant part of the semiannual density variations is associated with changes in the neutral composition.

Semiannual variations in the neutral composition, produced by global circulation must however be accompanied by a semiannual, latitude dependent temperature component.

It will be the subject of this paper to discuss the latitude dependent aspects of the annual and semiannual variations quantitatively on the basis of a theoretical model.

\section{THEORY}

Figure 1 shows a very simplified block diagram for the thermosphere dynamics. It illustrates the links between the composition and gas temperature 


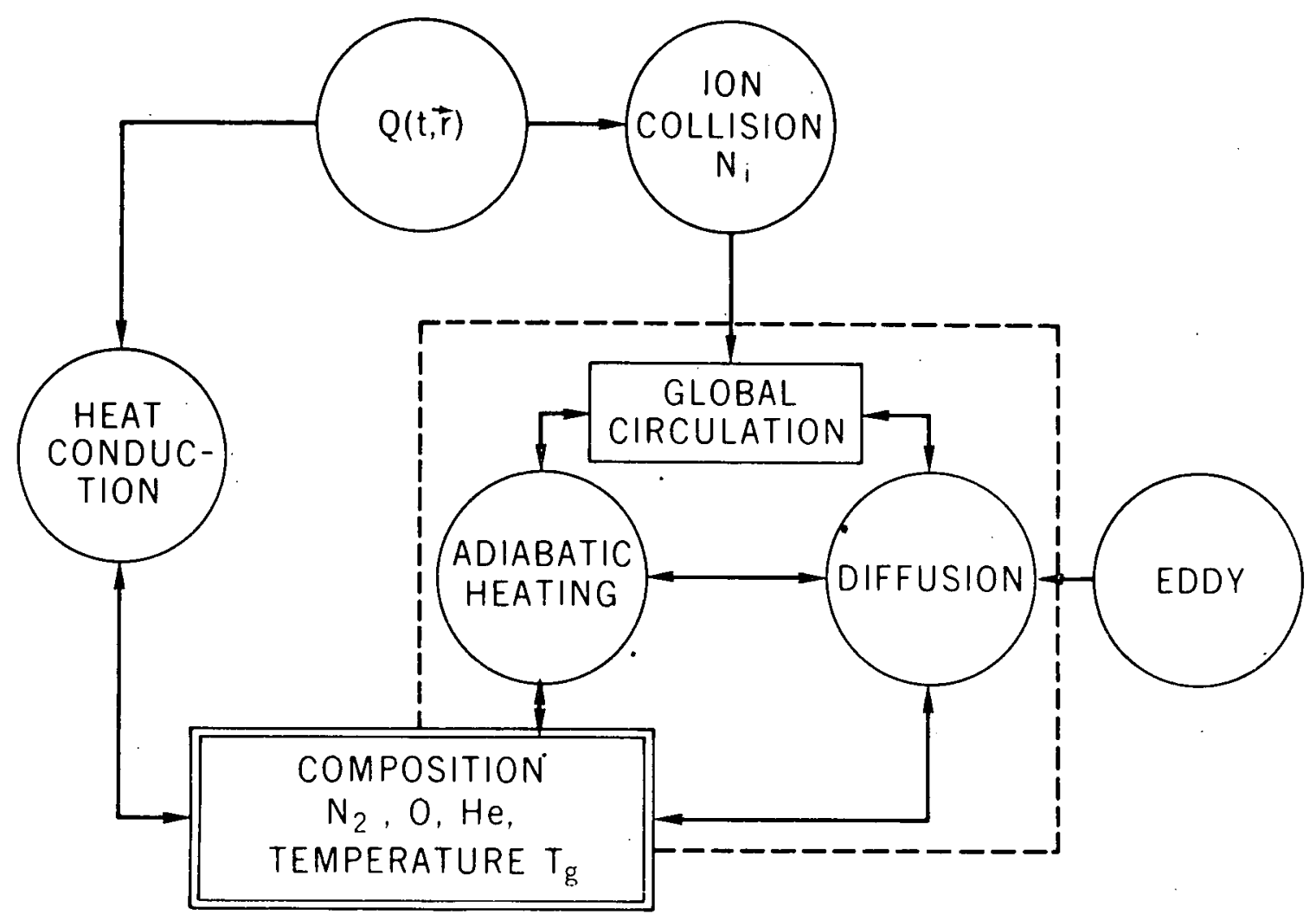

Figure 1. Schematic diagram for the physical processes that influence the temperature and composition of the thermosphere.

on one hand and the various physical processes and atmospheric parameters on the other hand.

Suppose the heat input $Q$ is known as a function of time, height, and latitude. Some of this energy input is conducted down into the lower atmosphere, thus affecting the temperature distribution and with that the composition.

However, a second and not less important energy train goes through the global circulation which is significantly affected by ion collisions and thus by the ion density. In this mechanism, which is explicitly neglected in one dimensional models, energy is exchanged between summer and winter through adiabatic expansion and contraction and this in turn affects the temperature and the thermospheric composition.

In parallel, the thermospheric circulation induces diffusion which depends upon the eddy coefficient, and this diffusion process affects the composition directly. Any variations in the composition are associated with variations in the 
total pressure field and this in turn influences the global circulation and with that adiabatic heating and with that again temperature and composition. So it is apparent that all the processes that have been surrounded by the dashed box in Figure 1 are inter connected. We shall show in this paper that the link between diffusion and energetics is extremely important for seasonal variations in the thermosphere.

To incorporate the diffusion processes in a self-consistent form, a two component model is adopted for the three dimensional thermosphere dynamics. With the subscripts 1 and 2 referring to the two atmospheric constituents, the equations of conservation of mass, momentum and energy are

$$
\begin{aligned}
& \frac{\partial \rho_{1}}{\partial \mathrm{t}}-O_{1}+\mathrm{L}_{1}+\frac{\partial}{\partial \mathrm{r}}\left(\rho_{1} \mathrm{~W}_{1}\right)+\operatorname{div}_{\theta}\left(\rho_{1} \overrightarrow{\mathrm{V}}_{1}\right)=0 \\
& \frac{\partial \rho_{2}}{\partial \mathrm{t}}-\mathrm{O}_{2}+\mathrm{L}_{2}+\frac{\partial}{\partial \mathrm{r}}\left(\rho_{2} \mathrm{~W}_{2}\right)+\operatorname{div} \mathrm{v}_{\theta}\left(\rho_{2} \overrightarrow{\mathrm{V}}_{2}\right)=0 \\
& \frac{\partial \mathrm{p}_{1}}{\partial \mathrm{r}}+\left(\frac{\mathrm{D} \mathrm{m}_{1}+\mathrm{K} \overline{\mathrm{m}}}{\mathrm{m}_{1}(\mathrm{D}+\mathrm{K})}\right) \mathrm{g} \rho_{1}+\phi \rho_{1} \rho_{2}\left(\mathrm{~W}_{1}-\mathrm{W}_{2}\right)=0 \\
& \frac{\partial p_{2}}{\partial r}+\left(\frac{D m_{2}+K \bar{m}}{m_{2}(D+K)}\right) g \rho_{2}+\phi \rho_{1} \rho_{2}\left(W_{2}-W_{1}\right)=0 \\
& \rho_{1}\left\{\frac{\partial \mathrm{v}_{1}}{\partial \mathrm{t}}+\nu_{1} \mathrm{v}_{1}+\phi \rho_{2}\left(\mathrm{v}_{1}-\mathrm{v}_{2}\right)+2 \Omega \cos \theta \mathrm{U}_{1}\right\}-\eta \frac{\partial^{2} \mathrm{v}_{1}}{\partial \mathrm{r}^{2}}=0 \\
& \rho_{2}\left\{\frac{\partial \mathrm{v}_{2}}{\partial \mathrm{t}}+\nu_{2} \mathrm{v}_{2}+\phi \rho_{1}\left(\mathrm{v}_{2}-\mathrm{v}_{1}\right)+2 \Omega \cos \theta \mathrm{U}_{2}\right\}-\eta \frac{\partial^{2} \mathrm{v}_{2}}{\partial \mathrm{r}^{2}}=0 \\
& \rho_{1}\left\{\frac{\partial \mathrm{U}_{1}}{\partial \mathrm{t}}+\nu_{1} \sin \mathrm{I} \mathrm{U}_{1}+\phi \rho_{2}\left(\mathrm{U}_{1}-\mathrm{U}_{2}\right)-2 \Omega \cos \theta \mathrm{V}_{1}\right\}-\eta \frac{\partial^{2} \mathrm{U}_{1}}{\partial \mathrm{r}^{2}}+\frac{1}{\mathrm{r}} \frac{\partial \mathrm{p}_{1}}{\partial \theta}=0 \\
& \rho_{2}\left\{\frac{\partial \mathrm{U}_{2}}{\partial \mathrm{t}}+\nu_{2} \sin ^{2} \mathrm{I} \mathrm{U}_{2}+\phi \rho_{1}\left(\mathrm{U}_{2}-\mathrm{U}_{1}\right)-2 \Omega \cos \theta \mathrm{V}_{2}\right\}-\eta \frac{\partial^{2} \mathrm{U}_{2}}{\partial \mathrm{r}^{2}}+\frac{1}{\mathrm{r}} \frac{\partial \mathrm{p}_{2}}{\partial \theta}=0
\end{aligned}
$$


$\frac{3}{2} k\left(\frac{\rho_{1}}{m_{1}}+\frac{\rho_{2}}{m_{2}}\right) \frac{\partial T}{\partial t}-\frac{\partial}{\partial r}\left(\kappa \frac{\partial T}{\partial r}\right)+p_{1} \frac{\partial W_{1}}{\partial r}+p_{2} \frac{\partial W_{2}}{\partial r}+p_{1} d i v_{\theta} U_{1}+p_{2} d i v_{\theta} U_{2}=Q(5)$

with

$$
\operatorname{div}_{\theta}=\frac{1}{\sin \theta} \frac{\partial}{\partial \theta}(\sin \theta)
$$

and

$\rho_{1}, \rho_{2}=$ mass densities

$W_{1}, W_{2}=$ vertical transport velocities

$v_{1}, v_{2}=$ horizontal transport velocities in the longitudinal direction

$\mathrm{U}_{1}, \mathrm{U}_{2}=$ horizontal transport velocities in the latitudinal direction

$\mathrm{r}, \theta=$ radial distance, colatitude

$\mathrm{O}_{1}, \mathrm{O}_{2}=$ chemical production terms

$\mathrm{L}_{1}, \mathrm{~L}_{2}=$ chemical loss terms

$\nu_{1}, \nu_{2}=$ collision frequencies with ions

$\phi=\frac{k T}{(D+K) m_{1} \rho_{1}}$

$\mathrm{k}=$ Boltzmann constant

$\mathrm{T}=$ temperature

$\mathrm{D}, \mathrm{K}=$ molecular eddy diffusion coefficient

$\mathrm{p}_{1}, \mathrm{p}_{2}=$ partial pressures

$\eta=$ viscosity coefficient

$\kappa=$ conductivity coefficient

$\mathrm{Q}=$ heat input rate 


$$
\begin{aligned}
& I=\text { dip angle } \\
& \Omega=\text { angular frequency of the earth's rotation. }
\end{aligned}
$$

In Equations (1) we have assumed the chemical production and loss terms to be zero in this paper, since we shall emphasize the dynamic aspects. In a subsequent paper we shall discuss the influences of $\mathrm{O}_{2}$ dissociation, recombination and the transport of chemical energy.

Equations (1) through (5) are solved under the following assumptions:

(1) The variables are separated into time t, latitude and altitude $\mathrm{r}$ components in the form

$$
\begin{aligned}
& \rho=\rho_{0}+\rho(r) P_{\rho}(\theta) e^{j \omega\left(t-t_{\rho}\right)} \\
& p=P_{0}+P(r) P_{p}(\theta) e^{j \omega\left(t-t_{p}\right)} \\
& T=T_{0}+T(r) P_{T}(\theta) e^{j \omega\left(t-t_{T}\right)} \\
& W=W(r) P_{W}(\theta) e^{j \omega\left(t-t_{W}\right)} \\
& V=V(r) P_{V}(\theta) e^{j \omega\left(t-t_{V}\right)} \\
& U=U(r) P_{U}(\theta) e^{j \omega\left(t-t_{U}\right)}
\end{aligned}
$$

where $\omega$ are the angular frequencies corresponding to the periods of one and one half years, and $t_{i}$ are the phases (times of maxima) for the individual atmospheric parameters. For the latitude dependences, $P_{\rho}, P_{p}$, etc., expansions into spherical harmonics are adopted thereby neglecting higher order terms:

$$
P_{\rho}=P_{p}=P_{T}=P_{W}= \begin{cases}P_{1}(\theta): & \text { annual } \\ P_{2}(\theta): & \text { semiannual }\end{cases}
$$


with

$$
\begin{aligned}
& P_{U}= \begin{cases}P_{0} \sin \theta: & \text { annual } \\
P_{1} \sin \theta: & \text { semi annual }\end{cases} \\
& P_{v}= \begin{cases}P_{0} \sin \theta \cos \theta: & \text { annua } 1 \\
P_{1} \sin \theta \cos \theta: & \text { semiannual }\end{cases}
\end{aligned}
$$

$$
\begin{aligned}
& \mathrm{P}_{0}=1 \\
& \mathrm{P}_{1}=\cos \theta \\
& \mathrm{P}_{2}=1 / 2\left(3 \cos ^{2} \theta-1\right)
\end{aligned}
$$

being Legendre polynomials. This simplification is made for mathematical convenience and in order to keep the computation time in limits that guarantee the usefulness of the model as a tool. It can be shown however that these functions approximate rather well the corresponding fundamental planetary waves at thermospheric heights (Volland and Mayr, 1971).

(2) Considering the functional forms (6) through (11) in the continuity equations (1) through (5), perturbation theory is applied. This leads (after differentiating some equations) in a straight forward manner to a set of 9 complex or 18 real second order differential equations which will not be shown here in the interest of conciseness. These equations describe the height dependence in the amplitude and phases of the atmospheric parameters.

The numerical scheme in performing the integration is essentially that of Lindzen and Kuo (1969). It will be seen in section 4 that in the case of the annual wave the amplitudes of $\mathrm{N}_{2}$ and of He become quite large so that the validity of perturbation theory may be questionable. However these amplitudes become greater than one primarily in those heights where $\mathrm{N}_{2}$ and He are the minor constituents. Therefore they cannot influence significantly the energetics and dynamics of the total gas. Amplitudes greater than one are of course not realistic in a linear theory and should be considered as upper limits.

The boundary conditions employed in the calculations where the follwing:

(1) At the lower boundary it was assumed that the characteristic time of the atmosphere is so long that no significant variations can be excited with the seasonally varying energy input. Accordingly we assumed 


$$
\rho=\mathrm{p}=\mathrm{T}=\mathrm{W}=\mathrm{U}=\mathrm{V}=0 \text { at } 80 \mathrm{~km} \text {. }
$$

This assumption is strictly speaking somewhat arbitrary, and therefore the solution is meaningless at the lower boundary level. It can be shown however that at higher altitudes above $120 \mathrm{~km}$ the solution can be considered as unique.

(2) Because of the low internal energy and heat input rate it was assumed that at the upper boundary the transport of mass, energy and momentum have negligible effects upon the thermosphere dynamics. It was therefore postulated that

$$
\frac{\partial V}{\partial r}=\frac{\partial U}{\partial r}=\frac{\partial T}{\partial r}=0 \text { at } 50 \dot{0 m}
$$

Again it must be noted that this assumption is actually not completely valid. The effect from this ambiguity, however, becomes negligible below $400 \mathrm{~km}$.

\section{INPUT PARAMETERS}

For the heat input due to the solar radiation a height dependence

$$
\mathrm{Q}=\mathrm{Q}_{0} \mathrm{e}^{-\left(\mathrm{r}-\mathrm{r}_{0}\right) / \mathrm{H}} \quad\left(\mathrm{r} \geqq \mathrm{r}_{0}=120 \mathrm{~km}\right)
$$

is adopted. Choosing a scale height of $\mathrm{H}=50 \mathrm{~km}$ we assume the same distributions that has been used in our model for the diurnal variations in the neutral composition (Mayr and Volland, 1972).

From a harmonic analysis of the solar heat input into the atmosphere (Volland and Mayr 1971) the amplitudes of the annual and semiannual components are approximately related to the diurnal component $Q_{0}^{D}$ through the relations

$$
\begin{gathered}
Q_{0}^{A}=0.4 Q_{0}^{D} \\
Q_{0}^{S A}=-0.075 Q_{0}^{D}
\end{gathered}
$$


where $Q_{0}^{A}$ and $Q_{0}^{S A}$ are the coefficients for the latitude dependences $P_{1}^{0}(0)$ and $\mathrm{P}_{2}^{0}(\theta)$ in the annual and semiannual heat input distributions, respectively.

The actual heat input distribution in the thermosphere is quite uncertain. For this reason $Q_{0}^{D}$ has been chosen as a free parameter which was determined in Mayr and Volland (1972) to

$$
Q_{0}^{D}=2 \times 10^{-7} \mathrm{ergs} / \mathrm{cm}^{3} \mathrm{sec} \text { at } 120 \mathrm{~km}
$$

such that the resulting temperature amplitude agreed with observations. Correspondingly we shall choose in this paper the values

$$
\begin{gathered}
Q_{0}^{A}=8 \times 10^{-8} \mathrm{erg} / \mathrm{cm}^{3} \mathrm{sec} \\
\mathrm{Q}_{0}^{\mathrm{SA}}=-1.5 \times 10^{-8} \mathrm{erg} / \mathrm{cm}^{3} \mathrm{sec} .
\end{gathered}
$$

The input (16a) will be the only source for the annual variations in our model. For the semiannual component, however, the source (16b), which is due to the sun's semiannual migration across the equator, is only one contribution.

In addition to (16b) at least the contribution from the semiannual component in the occurrence of magnetic storms must be considered.

For the electron density, which enters into the collision frequencies $\nu_{1}$ and $\nu_{2}$ an altitude distribution of the form

$$
N_{e}=N_{m} e^{\left(r-r_{m}\right) / H(r)}
$$

is used, with $H(r)$ varying in a form

$$
H(r)=h_{1}\left(r-r_{m}\right)+h_{2}\left(r-r_{m}\right)^{2}
$$

such that the scale height becomes 


$$
\mathrm{H}_{1}=80 \mathrm{~km} \text { at } 120 \mathrm{~km} \text {. }
$$

and

$$
\mathrm{H}_{2}=-150 \mathrm{~km} \text { at } 400 \mathrm{~km} \text {. }
$$

For the $\mathrm{F}_{2}$ maximum electron density a value of

$$
\mathrm{N}_{\mathrm{m}}=6 \times 10^{5} / \mathrm{cm}^{3}
$$

is adopted at $r_{m}=300 \mathrm{~km}$. With the neutral-ion drag coefficient of

$$
\xi=7.5 \times 10^{-10} \mathrm{~cm}^{3} / \mathrm{sec} \text { (Dalgarno, 1964), }
$$

the collision frequency $\nu_{1}=\nu_{2}=\nu$ is derived from

$$
\nu=\xi N_{e}\left(\frac{\omega_{L}^{2}}{\omega_{L}^{2}+\xi^{2} n^{2}}\right)
$$

where $\omega_{\mathrm{L}}=1.15 \times 10^{2} / \mathrm{sec}$ is the Larmor frequency for ions and $\mathrm{n}$ is the time average number density of the neutral atmosphere. The electron density is assumed to be latitude independent, and the expressions $\sin ^{2} \mathrm{I}_{1,2}$ in (4a) and (4b) are approximated by

$$
\sin ^{2} \mathrm{I} \mathrm{U}_{1,2} \sim \begin{cases}0.413 \mathrm{U}_{1,2}: & \text { annual } \\ 0.69 \mathrm{U}_{1,2}: & \text { semi annual }\end{cases}
$$

For the Coriolis force in (4a) and (4b) the following approximations were necessary in order to facilitate the separation into spherical harmonics:

$$
\begin{aligned}
& \cos ^{2} \theta \sim 0.333 \mathrm{P}_{0}^{0}: \text { annual } \\
& \cos \theta \mathrm{P}_{2}^{0} \sim 0.4 \quad \mathrm{P}_{1}^{0}: \quad \text { semi annual }
\end{aligned}
$$


The heat conductivity is computed from the function

$$
\kappa=5.25 \times 10^{3} \mathrm{~T}^{1 / 2} / \mathrm{M}
$$

with $M$ being the mean molecular mass in AMU. This form is in close agreement with the conductivity coefficients for the individual atmospheric constituents quoted in Harris and Priester (1962).

For the viscosity coefficients a form

$$
\eta=\eta_{0} \mathrm{~T}^{1 / 2}
$$

is adopted with a value of $\eta_{0}=1.3 \times 10^{-5}$ from Nicolet (1960).

Because relatively little is known about the eddy diffusion, a height independent average value of

$$
\mathrm{K}=4 \times 10^{6} \mathrm{~cm}^{2} / \mathrm{sec}
$$

is taken for the diffusion coefficient from Colgrove et al., (1965). However, this value will be modified in order to illuminate the sensitivity of the solutions to the eddy diffusion.

For the molecular diffusion coefficient the function

$$
\mathrm{D}=\mathrm{D}_{0}\left(\mathrm{~T} / \mathrm{T}_{\mathrm{s}}\right)^{1.75}\left(\mathrm{p} / \mathrm{p}_{\mathrm{s}}\right)^{-1}
$$

is used where $T_{s}$ and $p_{s}$ are standard temperature and pressure, $p$ being the sum of the partial pressures for the major constituents.

From the work of Walker (1961) a value of $D_{0}=0.26$ was taken for the diffusion of oxygen through $\mathrm{O}_{2}$ and $\mathrm{N}_{2}$. Considering that the diffusion coefficient depends on the square root of the reduced mass for the collision partners, a value of $D_{H E}=0.50$ is adopted for helium diffusing through $\mathrm{O}$ and $\mathrm{N}_{2}$. 
For the time average temperature distribution above $120 \mathrm{~km}$ a Jacchia (1964) model is adopted with an exospheric temperature of $T_{\infty}=1050^{\circ} \mathrm{K}$, while below 120 $\mathrm{km}$ the temperatures are taken from CIRA (1965). For the densities at $120 \mathrm{~km}$ the values are taken from Jacchia (1964).

It is assumed that the time average densities are in diffusive equilibrium. Since over the entire altitude range a constant eddy diffusion coefficient is used the transition between mixing and diffusive separation is continuous in our model.

\section{ANNUAL VARIATIONS}

Figure 2 shows in schematic form the basic elements of our theoretical model for the annual variations. The solar heat input maximum in the summer hemisphere causes the gas to expand at the summer pole. As a result, a circulation cell forms in which kinetic energy is transported from the summer to the winter hemisphere thus damping the temperature amplitude that would develop in the absence of this advective energy loss. At the same time this thermospheric

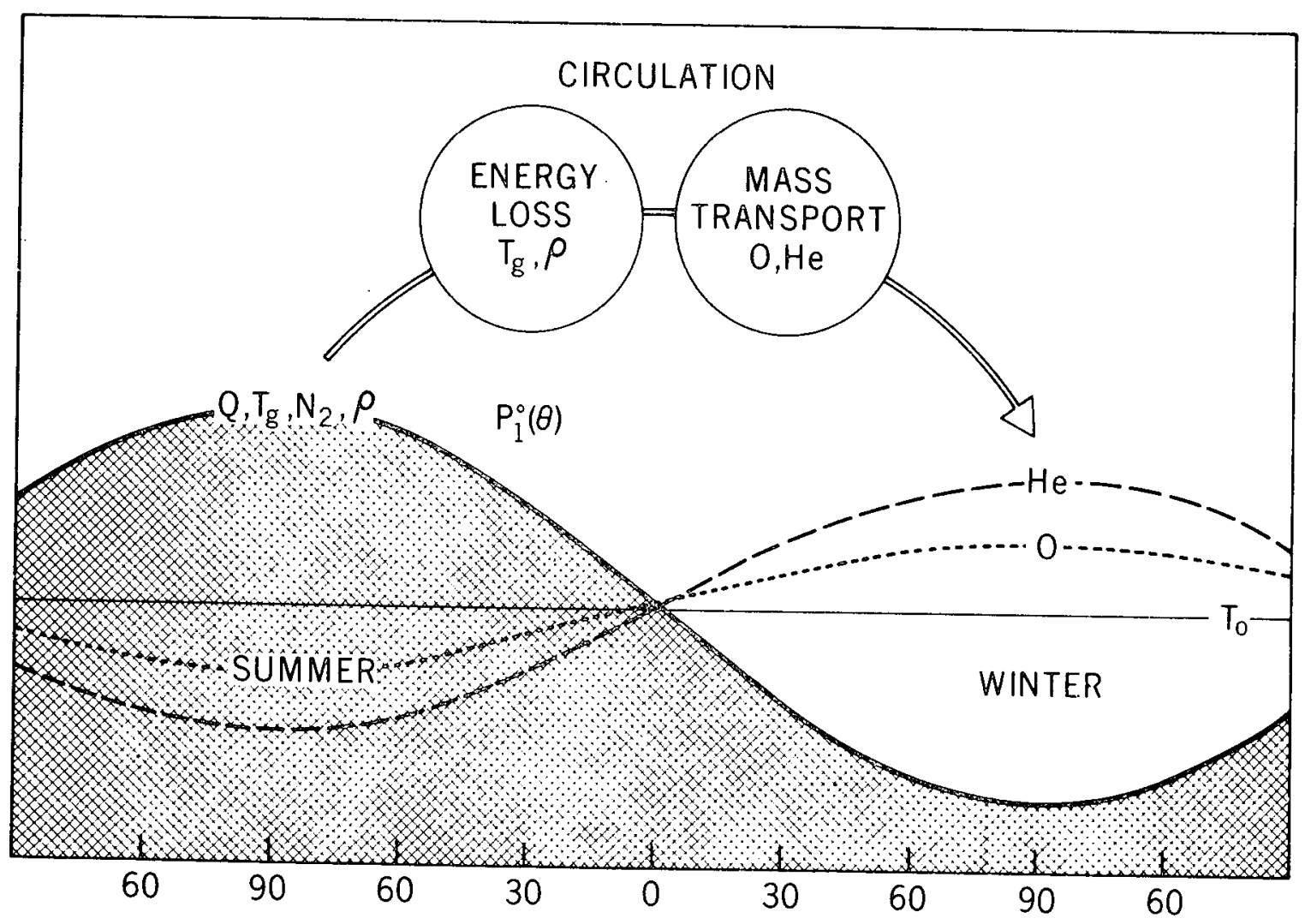

Figure 2. Schematic picture for the latisude dependence of the annual component of the various physical parameiters with the important processes emphasized in circles. 
circulation carries the minor and lighter constituents $\mathrm{O}$ and He from the summer to the winter hemisphere thus causing a redistribution in the thermospheric composition which is characterized by the $\mathrm{He}$ and $\mathrm{O}$ bulges in the winter hemisphere.

Figure 3 shows the amplitudes for $\mathrm{N}_{2}, \mathrm{O}, \mathrm{He}$, and $\mathrm{T}_{\mathrm{g}}$ at the summer pole from a solution of our theoretical models. With $\Delta \mathrm{m} \neq 0$, our $\mathrm{N}_{2}-\mathrm{O}$ model is identified. In this model the diffusion of both these constituents is considered and thus it represents also a realistic condition for the energetics of the thermosphere which is essentially determined in the region where $\mathrm{N}_{2}$ and $\mathrm{O}$ are the major constituents. With $\Delta \mathrm{m}=0$, our He-model is characterized in which the diffusion of He through $\mathrm{N}_{2}$ and $\mathrm{O}$ is treated, assuming that the mean molecular mass of these constituents combined does not vary as a function of time.

ANNUAL VARIATIONS

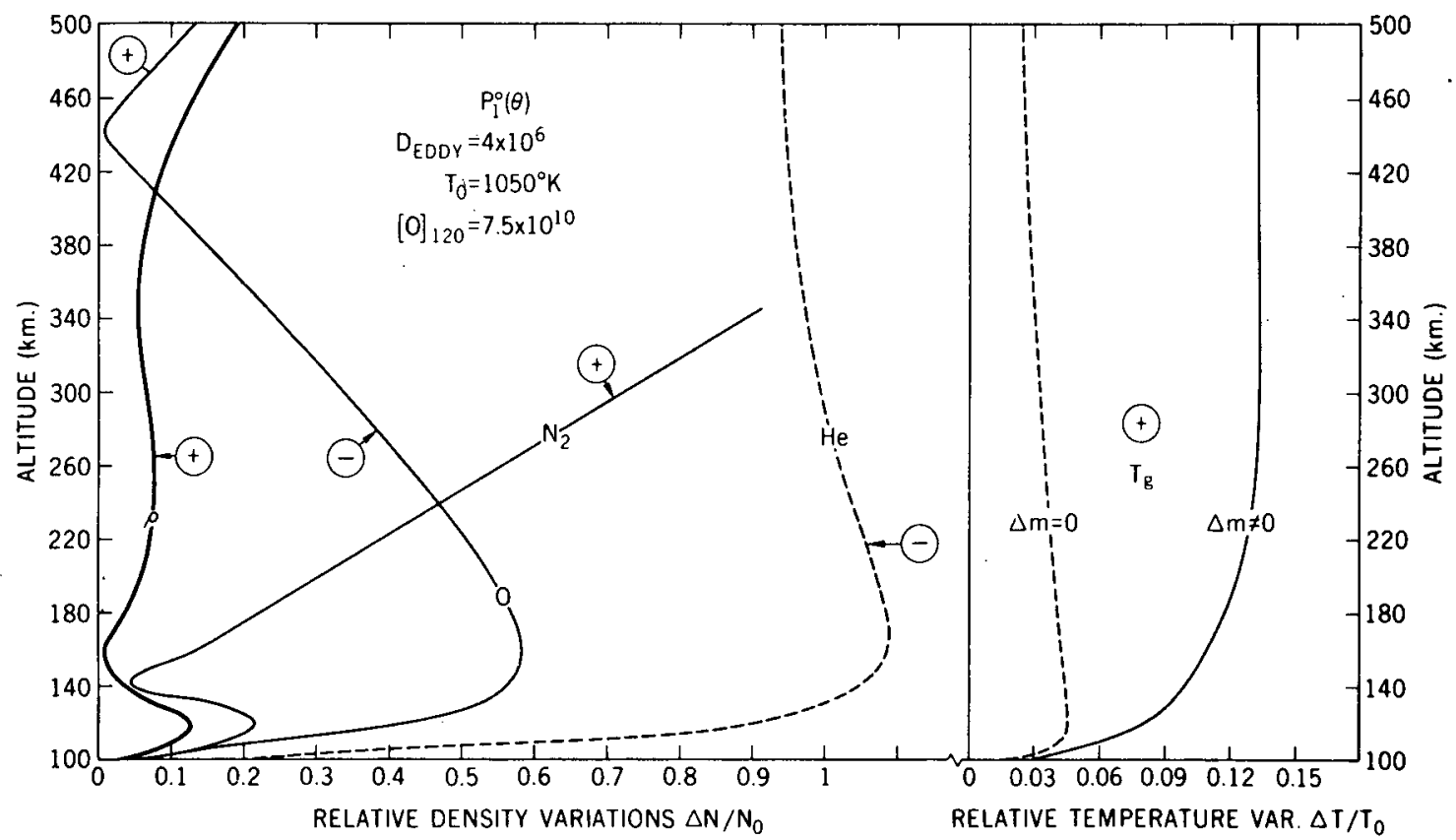

Figure 3. Relative amplitudes for the $\mathrm{O}-, \mathrm{He}-$, and $\mathrm{H}_{2}$-concentrations, the mass density $\rho$ and the gas temperature $T_{g}$ in the annual component. The values are valid for the summer pole and are the coefficients of the spherical function $P_{1}(\theta)=\cos \theta(\theta=$ polar distance) which describes the latitude dependence. Positive signs indicate positive, maximum values, negative signs indicate negative, minimum values in the northern summer hemisphere. Solid lines are taken from the $\mathrm{N}_{2}$-O-model which describes correctly the energetics and thus the gas temperature. Dashed lines stem from the He-model which is energetically unrealistic corresponding to the assumption of diffusive equilibrium for the major constituents $\mathrm{N}_{2}$ and $\mathrm{O}$ up to $500 \mathrm{~km}$. Note the large difference between the "diffusive equilibrium" solution and the "diffusion" solution for the gas temperature $\mathrm{Tg}$ as well as the small and nearly height independent amplitude of the mass density $\rho$. 
With plus signs, it is indicated that a particular atmospheric parameter is in phase with the solar heat input or that it increases in the summer and decreases in the winter hemisphere, while the minus signs indicate a decrease in the summer and an increase in the winter hemisphere.

From this solution it is then apparent that a large winter helium bulge develops throughout the entire altitude range above $100 \mathrm{~km}$. This result is basically in agreement with (a) the observations of Keating and Pior (1968) who detected the winter helium bulge in the satellite drag data and (b) the theoretical results of Johnson and Gottlieb (1970) who adopted a more or less reasonable wind field for their calculations.

The relative temperature amplitude in our He solution is about $4 \%$ and thus very close to the $5 \%$ variation inferred from the annual component in the satellite drag data (Jacchia, 1965). This kind of agreement is very significant because our He solution corresponds essentially to one of diffusive equilibrium for the major constituents up to $500 \mathrm{~km}$, a condition also presumed to be valid in Jacchia's model. However, deviating from Jacchia's model which assumes that the temperature variations are zero at $120 \mathrm{~km}$, our results show there a relatively large temperature amplitude. And this result had to be expected considering that the response time of the thermosphere is very much shorter than the period of a year even within the lower ther mosphere, a situation quite different from those encountered in the diurnal and magnetic storm variations.

With solid lines the solution from our $\mathrm{N}_{2}-\mathrm{O}$-model is represented. Since in this model the diffusion of the major constituents $\mathrm{O}$ and $\mathrm{N}_{2}$ is considered in a self consistent form, it provides a realistic picture for both the $\mathrm{O}-$ and $\mathrm{N}_{2}-$ composition as well as for the gas temperature.

This solution shows the formation of a winter oxygen bulge which is particularly pronounced within the lower thermosphere. It confirms thus the results by Mayr and Volland (1971) which were based on a wind field inferred from meteor trail observations. With a factor of three increase in $O$ between summer and winter pole at $120 \mathrm{~km}$, the formation of the winter anomaly in the $F_{2}$ region can thus easily be tied to the global circulation as has been suggested by Johnson (1964) and King (1962).

Although the diffusion velocities are generated within the entire thermosphere, they are effective primarily within the lower thermosphere and mesosphere where the ambient density is relatively high. Thus at higher altitudes the thermal expansion, associated with the temperature peak in the summer hemisphere, becomes increasingly more important. The consequence is that in the summer hemisphere the depletion of atomic oxygen, associated with the global circulation, becomes more and more compensated by the thermal expansion at 
higher altitudes. This is apparent in Figure 3 where the oxygen distribution exhibits an isopicnic level at exospheric heights. Below this level, the diffusion process dominates, and an oxygen bulge develops in the winter hemisphere; above it, the oxygen density is in phase with the temperature and thus peaks in the summer hemisphere. In helium, which is even more sensitive to the diffusion process and relatively insensitive to the temperature variations, such an isopicnic level can not develop and thus the winter helium bulge remains intact throughout the ther mosphere.

One of the major results that comes out of our model is that the diffusion process has a very strong effect upon the temperature amplitude. With the depletion and enrichment of oxygen in the summer and winter hemispheres, respectively, (induced by the diffusive redistribution) the total pressure variation in the annual component is substantially damped. Hence also the meridional velocities are significantly reduced and with that the adiabatic energy exchange, the consequence being that a much larger temperature variation can be maintained. In a way the diffusion process constitutes an effective viscosity which damps the meridional velocities and thus tends to isolate the solar heat input. In the $\mathrm{N}_{2}-\mathrm{O}$-model (solid line) which is appropriate for describing the temperature distribution, the amplitude is about $13 \%$ at exospheric heights thus about three times larger than that from the He-model (dashed line) in which the diffusion of the major constituents has not been considered. This temperature amplitude is also by about a factor of three greater than the temperature amplitude in the annual component of Jacchia's model.

A temperature amplitude of this magnitude, if associated with a diffusive equilibrium model, would produce meridional velocities of such magnitude that the corresponding energy exchange would essentially wipe out the temperature variations. It must be emphasized that such a large temperature amplitude can only be understood in the framework of a model in which diffusion and adiabatic heating are considered in a self-consistent form.

Variations in the total mass density are finally shown as a thick solid line in Figure 3, the plus sign indicating that, as expected, the density increases in the summer hemisphere. This quantity which is essentially observed in the satellite drag data reflects the superposition of two effects that tend to cancel each other: the thermal expansion of $\mathrm{N}_{2}$ and the depletion of $\mathrm{O}$ in the summer hemisphere (and vice versa in the winter hemisphere). In this respect the mass density has thus a characteristic quite similar to that of the total pressure variation which determines the wind field. It is apparent then from Figure 3 that the amplitude in the density is relatively small with some tendency even to decrease between 260 and $350 \mathrm{~km}$, the latter reflecting upon the increasing relative abundance of atomic oxygen at these heights. 
Although the details in the height distribution of the mass density depend on a number of factors to be discussed in the following, it is characteristic for its amplitude that it tends to remain constant with height. An interpretation of the satellite drag data in terms of a diffusive equilibrium model would therefore lead to the conclusion that this height structure is produced by a wave that travels from the lower atmosphere up through the thermosphere; while in reality we just see the manifestation of a highly variable composition which in fact is not in diffusive equilibrium.

In Figure 4 the annual component of the thermospheric wind field is presented from the $\mathrm{N}_{2}-\mathrm{O}$-model. The maximum vertical velocities, $\mathrm{W}$, are directed upward and are shown for the summer pole, the maximum meridional velocities, $U$, blowing toward the winter hemisphere are shown at the equator, and in the northern hemisphere the zonal components, $V$, are eastward in winter and westward in summer with their maximum magnitudes taken at $45^{\circ}$ latitude. The zonal wind
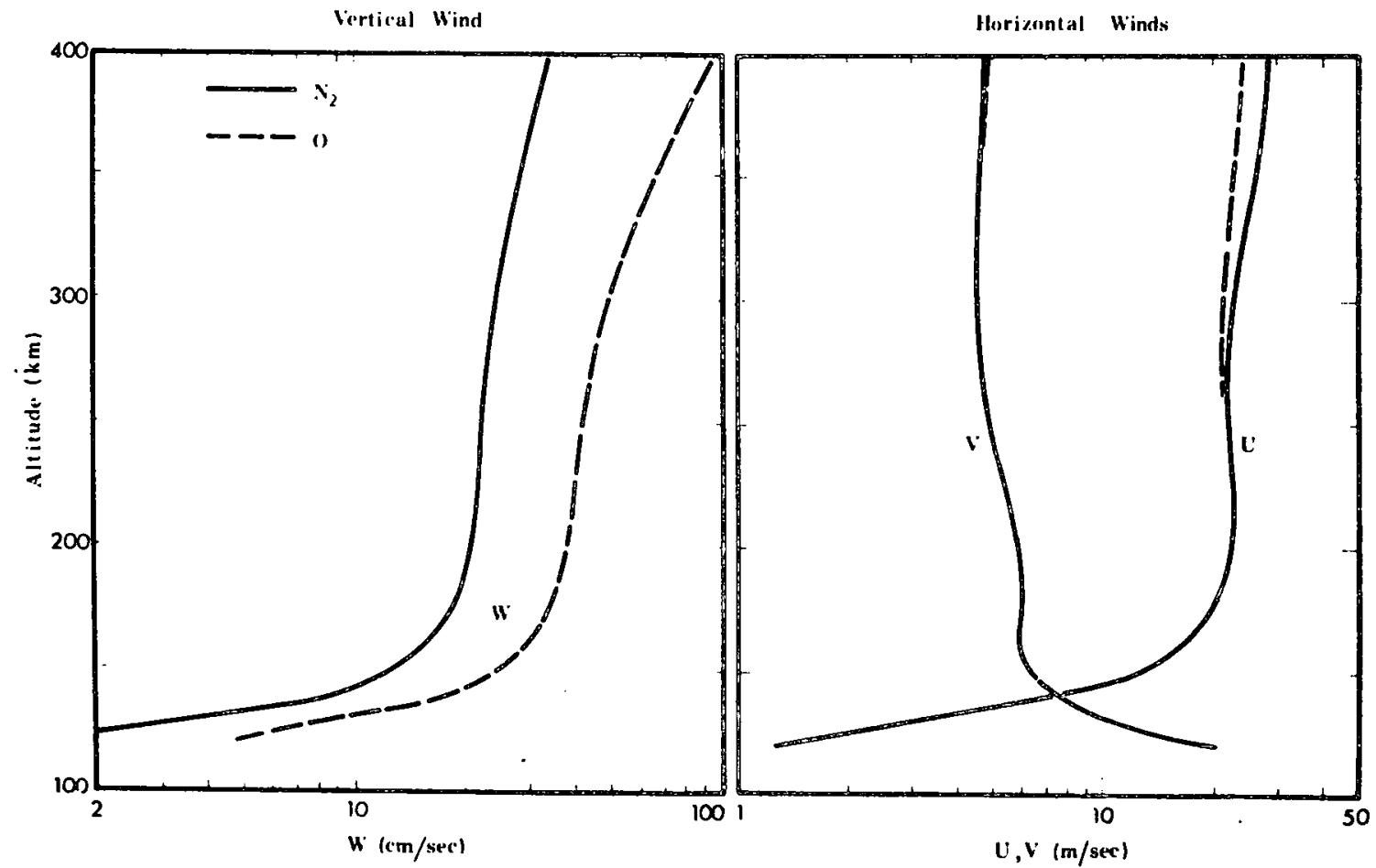

Figure 4. Amplitudes from the thermospheric wind field in the annual component of the $\mathrm{N}_{2}-\mathrm{O}$ model. The maximum vertical velocities, $W$, are direcied upward and are shown for the summer pole, the maximum meridional velocities, $U$, blowing toward the winter hemisphere are shown af the equator, and in the northern hemisphere the zonal winds, $V$, are eastward in winter and westward in summer with their maximum amplitudes at $45^{\circ}$ latitude. Dashed lines are for the $0-\mathrm{com}$ ponent, solid lines are for the $\mathrm{N}_{2}$-component. Note that the difference between both constituents is particularly large for $W$, but is al so not negligible for $U$. 
which is due to the geostrophic component of the wind field decreases toward higher altitudes thus indicating the diminishing influence of the Coriolis force. The meridional wind velocity increases with height reflecting the increasing importance of ion drag and viscosity which produce winds parallel to the pressure gradient. The magnitudes of both wind components are consistent with the results from a three dimensional theoretical model (Volland and Mayr, 1971).

It is apparent that the horizontal transport velocities for $\mathrm{O}$ and $\mathrm{N}_{2}$ can be different by as much $25 \%$ at exospheric heights. Therefore the diffusion effect of the horizontal wind field can not be entirely neglected as this is possible for the diurnal variation.

Much more significant is the difference between the vertical transport velocities of both constituents with the oxygen velocity being by about a factor of two larger than that of $\mathrm{N}_{2}$. It is this difference which accounts for the decrease in the scale height of atomic oxygen during summer and its increase during winter.

The eddy diffusion properties of the mesosphere are not well known. For this reason it is of interest to discuss the influence of eddy diffusion upon the thermospheric structure. In Figure 5 two solutions are presented from the $\mathrm{N}_{2}$ O-model for eddy diffusion coefficients of $2 \times 10^{6}$ and $8 \times 10^{6} \mathrm{~cm}^{2} / \mathrm{sec}$. From Figure 5 it is apparent that all atmospheric quantities are significantly affected. The most drastic effect occurs in the amplitude of the oxygen concentration which increases by almost a factor of two at $120 \mathrm{~km}$ height when the eddy diffusion coefficient has increased by a factor of four. Since the relative variations of $O$ are primarily responsible for the temperature amplitude as pointed out before, the increase of the eddy diffusion coefficient also increases the temperature amplitude significantly. This in turn leads to a stronger compensation of the oxygen depletion by the superimposed thermal expension with the final effect that at higher altitudes the variation of the total density due to variations in the eddy diffusion becomes damped.

Similar effects are apparent when the total content of atomic oxygen is varied. E.g., an increase of $(\mathrm{O})$ at $120 \mathrm{~km}$ from $7.5 \times 10^{10} \mathrm{~cm}^{-3}$ to $1.5 \times 10^{11}$ $\mathrm{cm}^{-3}$ leads to a substantial reduction ( $\left.\sim 30 \%\right)$ of the relative variation of $O$ and $\mathrm{T}_{\mathrm{g}}$. With the uncertainties in the eddy diffusion coefficients and in the oxygen concentration the model is thus far from being unique and is therefore only considered a means of identifying some physical aspects of the thermosphere dynamics.

\section{SEMIANNUAL VARIATION}

A number of sources have been proposed for the excitation of the semiannual effect in the thermosphere. 


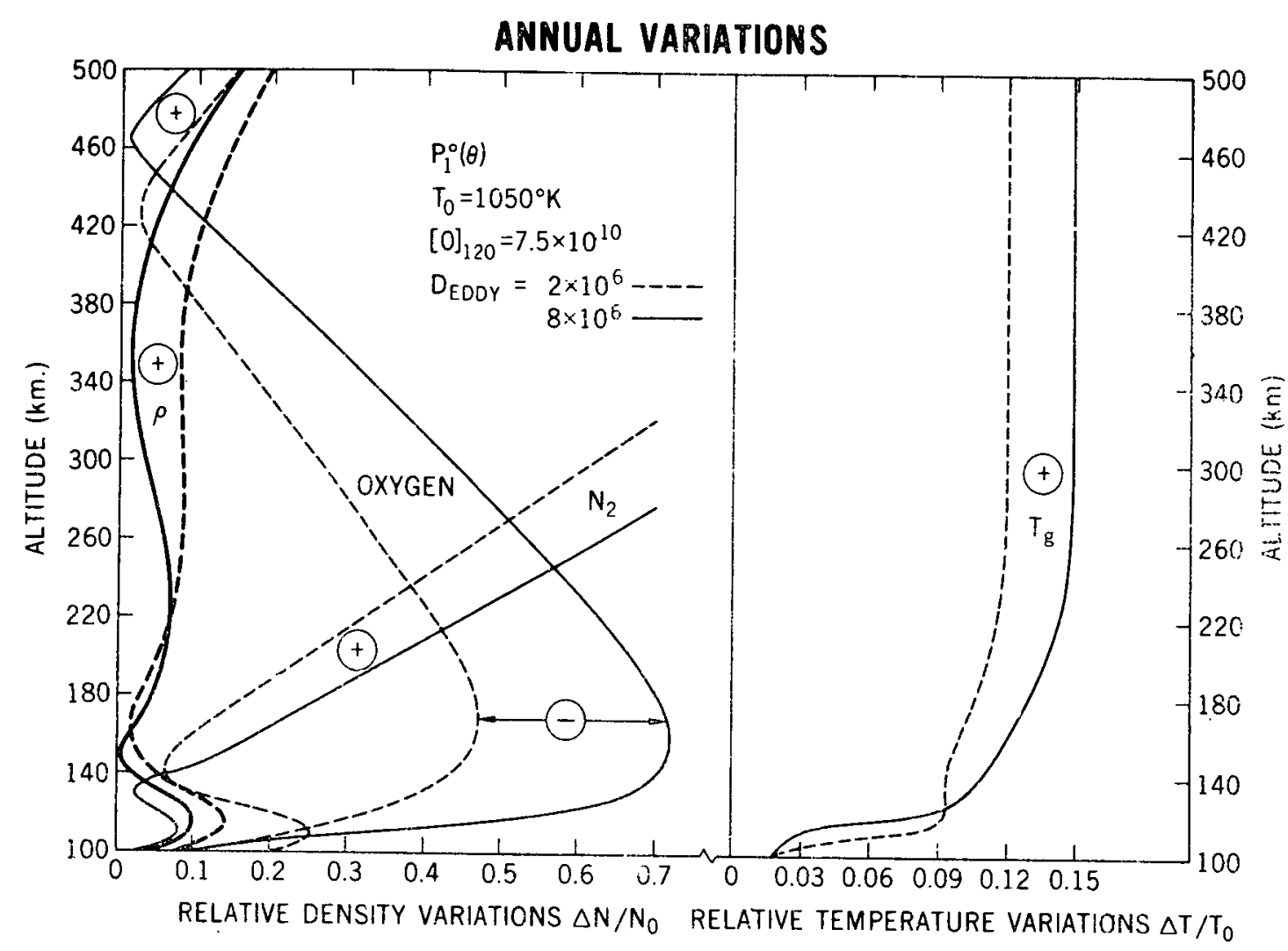

Figure 5. The $\mathrm{N}_{2}-\mathrm{O}$-model shown for different eddy diffusion coefficients. Note that all parameters are affected including $\mathrm{Tg}$.

(a) the non-linear energy coupling between the annual and semiannual variations,

(b) the solar EUV-component $(\mathrm{Eq}, 15)$ which is in a certain relation to the annual heat input,

(c) the auroral heat input associated with the semiannual component in the occurrance of magnetic storms and

(d) the energy coupling from the lower atmosphere where the semiannual effect is relatively strong.

We performed calculations on the non-linear coupling from the annual to the serniannual variations associated with adiabatic cooling and found it to be negligibily small at least for the latitudes dependent part of the semiannual variation. This result may be surprising in view of the relatively large variations in all atmospheric parameters of the annual component. Howeve: in the energy coupling the sum of both density components $\mathrm{O}$ and $\mathrm{N}_{2}$ enters, and as shown in section 4 , 
these quantities tend to cancel each other as is apparent from the small amplitude in the mass density in Figure 4.

It has been suggested by Mayr and Volland (1971) that a latitude dependence in the semiannual variation of the temperature and composition should be primarily excited by the magnetic storm component at high latitudes and by a much weaker source at low latitudes associated with the solar EUV heat input. Recent observations by Hedin et al., (1972) confirm this concept except with regard to the low latitude structure which could very well be masked by the dominance of the height latitude source.

We shall adopt here the simplified model that the latitude dependence in the semiannual variation is in a first approximation excited by a source of the form

$$
Q^{s a}=Q_{0}^{s a} P_{2}(\theta)
$$

which peaks at the poles during equinox reflecting the dominance of auroral heating. Since the magnitude of the magnetic storm component is not known, $\mathrm{Q}_{0}^{3 \mathrm{a}}$ is considered as a free parameter to be determined by matching the theory with the exospheric temperature derived from the data of Hedin et al., (1972). This value is

$$
Q_{0}^{s \theta}=2 \times 10^{-8} \mathrm{erg} / \mathrm{cm}^{3} \mathrm{sec} \text { at } 120 \mathrm{~km} \text { height, }
$$

which is of the same order as the heat input due to EUV (Eq. 16b), however reversed in sign. For the height dependence of $Q^{\text {sa }}$ we adopted the same formula as for in (14) with $\mathrm{H}=50 \mathrm{~km}$.

The results of our model calculations are shown in Figure 6. Again with dashed lines the He-model and with solid lines the $\mathrm{N}_{2}$-O-model is represented. The thick solid line gives the total mass density of the $\mathrm{N}_{2}-\mathrm{O}$ model. The amplitudes are the coefficients of the $\mathrm{P}_{2}$-function. They therefore describe the actual values at the poles. According to the dependence of $P_{2}$ on colatitude $\theta$ (see Eq. 11) plus-signs indicate that a particular quantity is enhanced at the poles and decreased at the equator during equinox. Minus signs indicate a decrease at the poles and an increase at the equator.

The features apparent from Figure 6 are completely analogous to those for the annual component in Figure 3. The thermospheric circulation that is produced by the equinoctial pressure and temperature maxima at the poles induces 


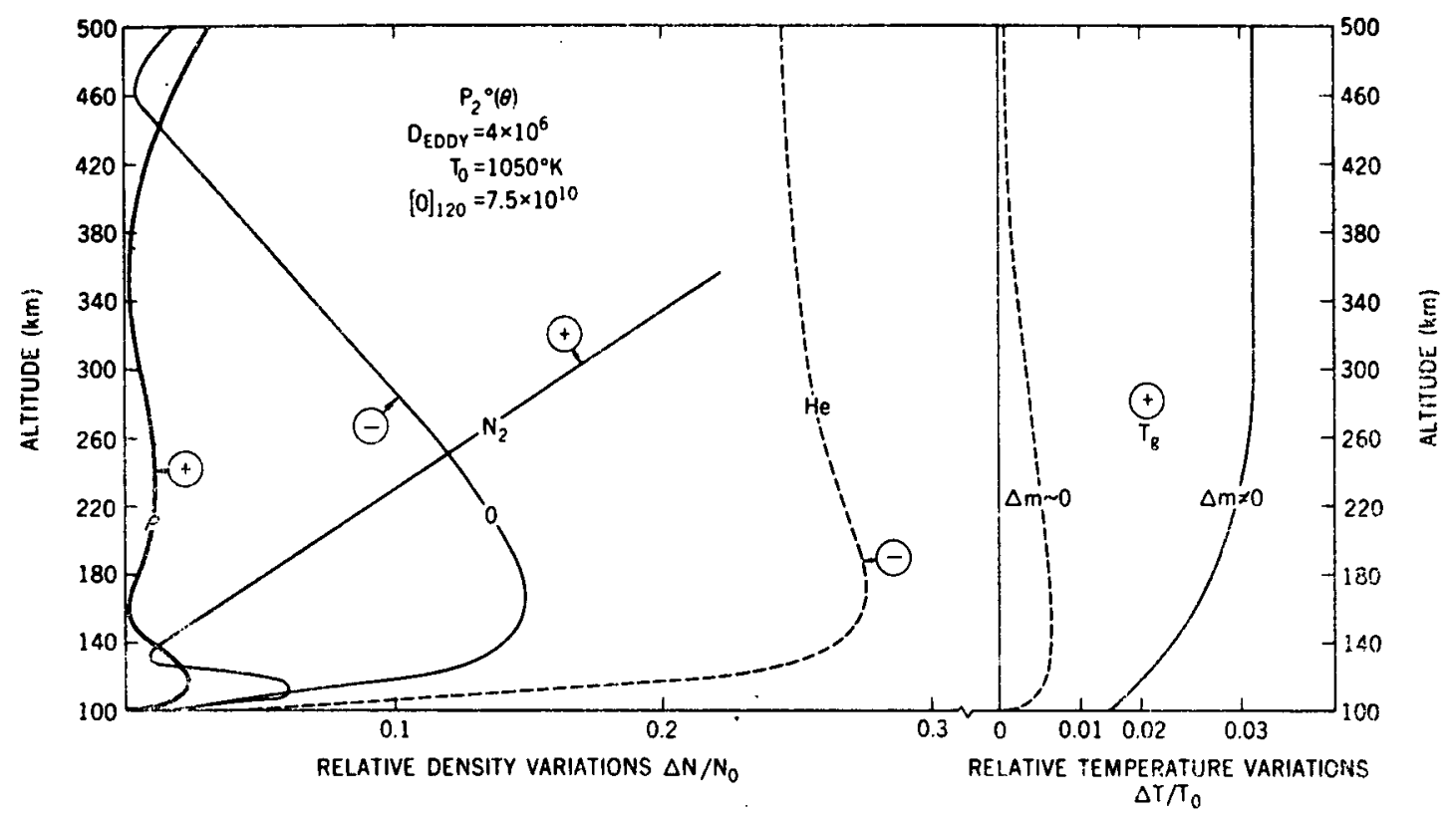

Figure 6. Relative amplitudes for the physical parameters of density and temperature of the semiannual variation. The values are validfor the poles and are the coefficients of the spherical harmonic $\mathrm{P}_{2}(\theta)$. Positive signs indicate at the poles maximum and minimum values during equinox and solstice, respectively. Negative signs indicate the opposite. The $\mathrm{N}_{2}-\mathrm{O}$-model and the He-model are differentiated with solid and dashed lines, respectively. Note the large difference between "diffusive equilibrium" solution (dashed line) and "diffusion" solution (solid line) for $T_{g}$, and the height independent and very small amplitude of the mass density $\rho$.

Cinere a depletion in the population of oxygen and helium. This effect is primarily pronounced at low altitudes (minus signs for oxygen and helium). It is graduately compensated by the thermal expension in the distribution of $O$ such that an isopicnic level develops near $450 \mathrm{~km}$ with oxygen being in phase with the temperature at higher altitudes.

In helium the thermal expension is less effective, and an isopicnic level cannot develop up to very high altitudes due to the more pronounced depletion effect. Therefore He decreases throughout the termosphere in the region of enhanced temperature. All these features are observed by Hedin et al., (1972).

The difference between the temperature amplitudes from the He-model (dashed line) and the $\mathrm{N}_{2}-\mathrm{O}$-model (solid line) is again very large and thus reflects upon the importance of the diffusion process for the thermospheric energetics in the seasonal variations, an effect that has been discussed in detail for the annual component. Since $\mathrm{N}_{2}$ and $\mathrm{O}$ are the major constituents up to 500 $\mathrm{km}$, the temperature amplitude from the $\mathrm{N}_{2}-\mathrm{O}$-model (solid line) is considered 
as realistic. It is this quantity which has to be compared with the temperature derived by Hedin et al., (1972). The distribution of the total mass density is flat and its amplitude is very small similar to the annual variations. The latter accounts for the fact that the satellite drag data have so far not revealed a latitude dependence in the semiannual variation as predicted by Mayr and Volland (1971).

\section{CONCLUSION}

A three dimensional model of the thermosphere is presented in which energy and diffusive mass transport associated with the global circulation are considered in a self consistent form. It is shown that these processes play a major role in the thermosphere dynamics and thus account for a number of composition and temperature phenomena which are now being observed. In particular, the model describes

(1) In the annual component

(a) the helium and oxygen bulges in the winter hemisphere,

(b) the relatively large temperature variations (26\% between winter and summer poles) which exceed by a factor of three the temperature variations from the Jacchia model and which would be entirely impossible in a diffusive equilibrium model,

(c) the nearly height independent amplitude of the total mass density which is in our model the manifestation of a highly variable composition.

(2) In the semiannual component

(a) the significant latitude dependence in the composition with a depletion of oxygen and helium at the poles and a corresponding enhancement in the density of these constituents at the equator during equinox,

(b) the latitude dependence of the temperature with a $7 \%$ variation at the poles where the temperature peaks during the equinox,

(c) the negligibly small amplitude in the latitude dependence of the mass density which is responsible for the fact that the latitude dependence of this effect has so far eluded observations from satellite drag data. 
It is the self consistent treatment of the diffusion and energy transport mechanisms that accounts for the agreement between our model and the latitude dependent components of the seasonal variations now being observed. Although sophisticated with regard to the physics of the thermosphere dynamics, the model is mathematically however relatively primitive. The application of perturbation theory and the restriction to the first harmonics with their simplification in the description of ion drag and Coriolis force characterize the approximations. This becomes particularly evident in our calculations when amplitudes larger than one result in the model. These therefore should be regarded as upper limits. Finally the relatively simple energy models as well as the neglect of chemical production and loss terms are further simplifications which are very likely however masked by the uncertainties in the eddy diffusion coefficients and in the absolute content of atomic oxygen.

\section{ACKNOWLEDGEMENT}

The authors are indebted to Mrs. J. Liu, now with Consultants and Designers, Greenbelt, for her assistance in the computer analysis.

\section{REFERENCES}

Brinton, H.C. and Mayr, H. G.; Temporal variations of thermospheric hydrogen derived from in situ measurements, J. Geophys. Res. $\underline{76}, 6198-6201,1971$.

CIRA, COSPAR international reference atmosphere, North-Holland Publ. Comp., 1965.

Colegrove, F. D., Hanson, W. B., and Johnson, F. S.; Eddy diffusion and oxygen transport in the lower thermosphere, J. Geophys. Res. 70, 4931-4941, 1965.

Cook, G. E.; The large semiannual variation in exospheric density: a possible explanation, Planet. Space Sci. $15,627-632,1967$.

Cook, G. E.; The semiannual variation in the upper atmosphere: a review, Ann. Geophys. 25, 451-469, 1969.

Cook, G. E.; The semiannual variation in the upper atmosphere during 1967 and 1968, Planet. Space Sci. 18, 565-582, 1970.

Dalgarno, A.; Ambipolar diffusion in the F-region, J. Atm. Terr. Phys. 26, 939, 1964. 
Harris, I. and Priester, W.; Time dependent structure of the upper atmosphere, J. Atm. Sci. 19, 286-301, 1962.

Keating, G. M. and Prior, E. J.; The winter helium bulge, Space Res. 8, North-Holland Publ. Comp., 982-992, 1968.

King, G. A. M.; The dissociation of oxygen and high level circulation in the atmosphere, J. Atm. Sci. 21, 231-237, 1964.

Jacchia, L. G.; Static diffusion models of the upper atmosphere with empirical temperature profiles, Smithsonian Institution, Astrophysical Observatory, Special Report 170, Cambridge, Mass., 1964.

Jacchia, L. G.; Semiannual variation in the heterosphere: a reappraisal, J. Geophys. Res. 16 , 4602-4607, 1971.

Jacchia, L. G. and Slowey, J. W.; The shape and location of the diurnal bulge in the upper atmosphere, Space Res. 7, North-Holland Publ. Comp., 1077$1090,1967$.

Jacchia, L. G., Slowey, J. W., and Verniani, F.; Geomagnetic perturbations and upper atmospheric heating, Smithsonian Institution, Astrophysical Observatory, Special Report 218, Cambridge, Mass., USA, 1966.

Johnson, F. S.; Circulation at ionospheric levels, Report of the Southwest Center of Advanced Studies, Contract CW-II, 10531, 1964.

Johnson, F. S. and Gottlieb B.; Eddy mixing and circulation at ionospheric levels, Planet. Space Sci. 18, 1707-1718, 1970.

Lindzen, R. S. and Kuo, H. L.; A reliable method for the numerical integration of a large class of ordinary and partial differential equations, Mon. Wea. Rev. 97, 732-734, 1969.

Mayr, H. G. and Mahajan, K.; Seasonal variations in the F2-region, J. Geophys. Res. 76, 1017-1028, 1971.

Mayr, H. G. and Volland, H.; Semiannual variation in the neutral composition, Ann. Geophys. 27, 513-522, 1971.

Mayr, H. G. and Volland, H.; A two component model of the diurnal variations in the thermospheric composition, NASA-Document, X-621-72-233, 1972

Newell, R. E.; Semiannual variation in thermospheric density, Nature 217, 150, 1968. 
Nicolet, M.; The properties and constitution of the upper atmosphere, in J. R. Ratcliffe (ed): "Physics of the Upper Atmosphere," Academic Press, New York and London, 1960.

Volland, H.; A theory of thermosphere dynamics: Part II, Planet. Space Sci. 17, 1709-1724, 1969.

Volland, H. and Mayr, H. G.; A three dimensional model of thermosphere dynamics: Part III, NASA Document X-621-71-242, GSFC, Greenbelt, Md., USA, 1971.

Walker, R. E.; Measurement of the O-O diffusion coefficient, J. Chem. Phys. 31, 2196-2197, 1961. 